\title{
Mobilier en fin de vie
}

Le design au service des perspectives durables

Where Furniture Goes to Die. Designing for Sustainable Behaviour in a Practice

Perspective

\section{Marie Hebrok}

\section{OpenEdition \\ Journals}

Édition électronique

URL : https://journals.openedition.org/tc/7853

DOI : $10.4000 /$ tc. 7853

ISSN : 1952-420X

Éditeur

Éditions de l'EHESS

\section{Édition imprimée}

Date de publication : 31 octobre 2016

Pagination : 154-157

ISBN : 9782713225291

ISSN : 0248-6016

Référence électronique

Marie Hebrok, « Mobilier en fin de vie », Techniques \& Culture [En ligne], 65-66 | 2016, mis en ligne le 31 octobre 2016, consulté le 29 septembre 2022. URL : http://journals.openedition.org/tc/7853 ; DOI : https://doi.org/10.4000/tc.7853 


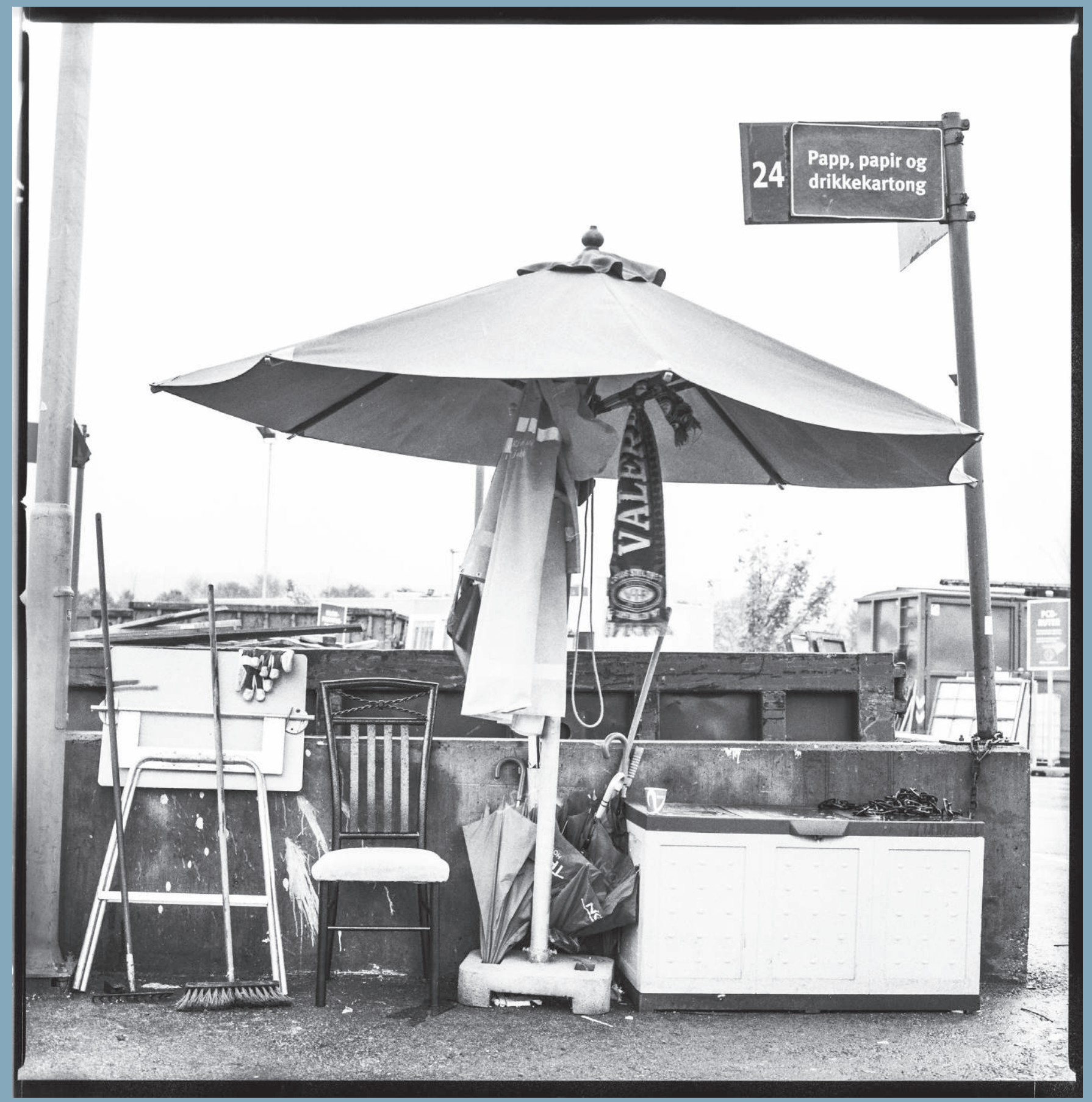




\section{Mobilier en fin de vie}

\section{Le design au service des perspectives durables}

Dans l'univers de la conception d'objets, des efforts sont en train d'être fournis pour augmenter la durée de vie des produits afin de réduire l'impact environnemental. Ce travail est le plus souvent axé sur la durabilité des matériaux, la «réparabilité », les constructions modulaires et les parties séparables de différents matériels dans une optique de recyclage. Au lieu de prolonger la période d'utilisation en essayant d'éviter ou de retarder leur élimination, des spécialistes en conception tendent à mettre l'accent sur la «recyclabilité» des matériaux et cherchent à créer des systèmes en circuit fermé où la matière n'est pas gaspillée, mais réintègre au contraire un cycle infini. En vue de réduire l'impact écologique de la production et de la consommation, ceci est important. Néanmoins, il s'agit de ce que Chapman appelle le «caractère intrinsèque de la conception durable axée sur les symptômes» (Chapman 2010 : 66). Bien que le fait d'avoir recours à davantage de matériaux et de méthodes de production durables soit mieux que rien, cela n'est aucunement comparable, en terme d'impact, aux mesures consistant à réduire le flux et la production de ces mêmes matériaux en prolongeant la durée de vie des produits.

Cet article examine l'utilité de la théorie de la pratique et propose une approche ethnographique de la méthodologie Design for a Sustainable Behaviour (DfSB) ou « design au service d'un comportement durable» en français. L'hypothèse consiste à inclure dans l'analyse concernant la mise au rebut des objets à la fois les aspects matériels et les aspects sociaux, sans accorder la primauté à l'un ou l'autre; les efforts dans le domaine de la conception pour prolonger la durée de vie des produits peuvent être plus fructueux. Les raisons qui poussent les personnes à se débarrasser de leur mobilier et comment ces informations peuvent être utiles aux concepteurs de meubles qui cherchent à développer des produits plus pérennes sont analysées ici. Dans la mesure où un meuble est en général un bien durable qui, grâce à un bon entretien, peut résister des siècles, les phénomènes d'usure et de pourriture ne constituent pas pleinement des raisons valables de le jeter. 
Les grands centres de recyclage situés en périphérie des villes où les gens peuvent se délester de leurs «affaires » en trop sont un bon observatoire de la mise au rebut. C'est l'endroit même où nos objets vont mourir, et les raisons qui sous-tendent leur fin ne sont pas toujours aussi évidentes que l'on croit. L'article suivant s'appuie sur trois visites du plus grand centre de recyclage d'Oslo où l'auteur a fait des observations et mené des entretiens. Son hypothèse repose sur le fait que les personnes peuvent révéler d'autres aspects de la mise au rebut lorsqu'elles sont abordées au moment précis de l'action et en présence de l'objet jeté, à la différence d'entretiens dans le cadre d'études et hors de l'acte.

Une théorie de la pratique est appliquée à l'analyse des raisons pour lesquelles les personnes jettent des meubles. Dans la perspective du DfSB, nous nous interrogeons également sur la possibilité d'influer, à travers le design, sur le comportement. L'objectif de cette étude est de prendre comme point de départ de l'analyse de la mise au rebut des meubles les pratiques sociales, afin de mieux comprendre comment les concepteurs de mobilier peuvent intervenir dans le prolongement de la durée de vie des produits. La conception du DfSB est basée sur l'idée selon laquelle le contrôle est réparti entre l'utilisateur et le produit, sur une échelle allant de l'utilisateur totalement contrôlé au produit totalement contrôlé. La problématique essentielle au développement de notre champ de recherches est la suivante: comment obtenir les connaissances indispensables, en matière d'action humaine, à l'orientation bénéfique du design afin de jouer sur l'utilisation des produits et les conséquences de leur utilisation, dans une perspective environnementale. La théorie de la pratique interroge les concepteurs et les chercheurs en conception en raison de la prise en compte, dans l'analyse, de l'aspect matériel.

Il existe un large éventail d'éléments de la pratique qui influent sur la mise au rebut des meubles. L'enjeu pour le designer est d'identifier à quel moment la conception de l'objet peut faire une différence dans la durée de vie de certains meubles. Cette approche a contribué à élaborer trois récits décrivant les éléments de pratiques liées à la mise au rebut du mobilier. Dans le but de prolonger ces récits jusqu'à envisager le moment de la conception concrète qui étendra la longévité des meubles et poussera les gens à adopter un mode de consommation plus pérenne, différentes stratégies issues des recherches en «design pour un comportement durable» sont avancées.

Cet article illustre la façon dont une telle analyse peut être menée sans pour autant se conformer à un modèle rigide, signifiant ainsi que le concepteur doit employer ses compétences d'analyse de façon à acquérir une plus grande compréhension de tous les éléments qui sont pertinents pour certains enjeux du DfSB. Il faut davantage recourir aux recherches appliquant les outils et les principes du DfSB à des cas réels. Des recherches plus poussées doivent être axées sur la conception actuelle des produits et s'appuyer sur les indices résultant de cette approche. En outre, les chercheurs en conception devraient s'efforcer d'étudier concrètement les effets actuels de l'application des principes du DfSB. Cette étude, bien que non exhaustive dans son procédé de mise en valeur de toutes les raisons pertinentes poussant les personnes à jeter du mobilier, souligne cependant certains des principaux enjeux apparus au concepteur. 
Cela est peut-être suffisant pour réaliser des conceptions qui sauront faire la différence, mais des vérifications s'imposent.

Toutefois, la faisabilité et l'utilité de mener une courte enquête de terrain semblent démontrées; un facteur important sachant que le temps c'est de l'argent. Dans le domaine des affaires, le DfSB pourrait être un concept inconnu pouvant influencer positivement sur les bénéfices. Pour ce faire, il est fondamental de trouver les bons domaines d'application de la recherche et du développement en conception, de même que les acteurs et les collaborateurs intéressés et souhaitant investir. Il existe bien entendu des limites à ce que peut contrôler le concepteur, et c'est là une des restrictions actuelles des démarches de conception.

\section{En ligne}

Retrouvez l'article complet, en version originale (anglais), sur revues.org, TechniquesECulture 65-66 « Réparer le monde. Excès, reste et innovation » : http://tc.revues.org.

\section{I'auteure}

Marie Hebrok est chargée de recherche à l'Institut national norvégien de la recherche sur la consommation (SIFO) à Oslo, au Collège universitaire de sciences appliquées d'Akershus (HiOA). Elle prépare actuellement une thèse de doctorat sur la réduction du gaspillage alimentaire à travers un « design au service d'un comportement durable » (Design for a Sustainable Behaviour). Elle est spécialisée dans la conception des produits, le design commercial, les recherches en sciences, technologie et innovation, ainsi que dans la recherche sur la consommation.

\section{Iconographie}

Image d'ouverture. Centre de recyclage d'Haraldrud. Le malheur des uns fait le bonheur des autres.

Crédits photographiques pour l'ensemble des images. (C) Erlend Berge.

\section{Pour citer cet article}

Hebrok, M. 2016 «Mobilier en fin de vie. Le design au service des perspectives durables », Techniques\& Culture 65-66 « Réparer le monde. Excès, reste et innovation »,

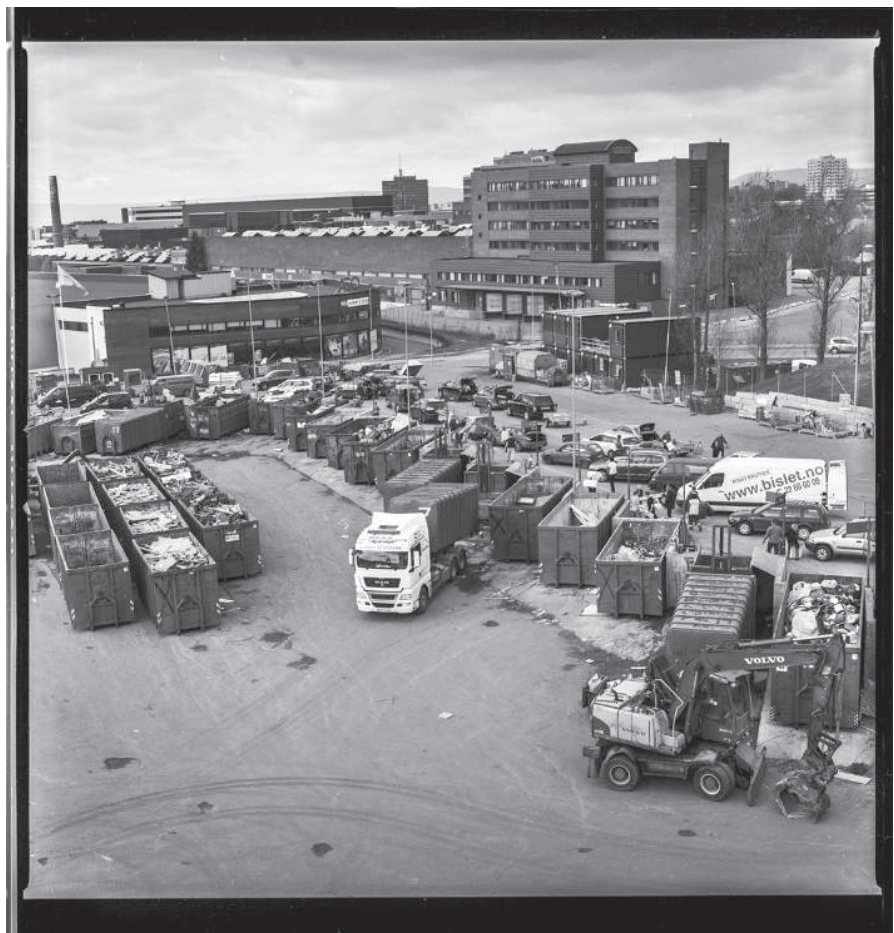

Centre de recyclage d'Haraldrud Tri des différents matériaux dans les containers. 